\title{
Representación del Proceso Formativo de una Institución Etnoeducativa a través de la Técnica del Modelo Gráfico y Descriptivo usando Diagramas de Influencia y de Forrester
}

\author{
Marlin A. Aarón ${ }^{(1)}$, Patricia E. Choles ${ }^{(2)}$ y Andrés D. Solano ${ }^{(3)}$ \\ (1) Facultad de Ingeniería, Grupo I+D Motivar. Universidad De La Guajira, Riohacha, Km 5 Salida a \\ Maicao. Colombia. (e-mail: maaron@uniguajira.edu.co) \\ (2) Facultad de Educación. Grupo I+D Motivar. Universidad De La Guajira, Riohacha, Km 5 Salida a \\ Maicao. Colombia. (e-mail: pcholes@uniguajira.edu.co) \\ (3) Miembro Grupo I+D Motivar. Univ. De La Guajira, Riohacha, Km 5 Salida a Maicao. Colombia. \\ (e-mail: andresolano@uniguajira.edu.co)
}

Recibido Oct. 6, 2015; Aceptado Nov. 26, 2015; Versión final Ene. 26, 2016, Publicado Jun. 2016

\begin{abstract}
Resumen
En este trabajo se describe un proceso formativo implementado en una institución etnoeducativa en una comunidad indígena en condiciones de marginalidad. La metodología que se usa para el desarrollo de este estudio es de corte cuali-cuantitativa para desarrollar el modelo descriptivo. Se emplea computación gráfica para construir los diagramas de influencia y el diagrama de Forrester. Estos permiten capturar gráficamente la esencia de este complejo sistema real y ayudan a comprender las relaciones presentes entre las variables del proceso formativo. La dinámica de sistemas visibiliza la influencia de variables independientes que perturban el proceso formativo tales como la ubicación geográfica, la fecha de contratación de los docentes y el transporte de los niños a la escuela.
\end{abstract}

Palabras clave: modelo gráfico; proceso formativo; diagrama de Forrester; diagrama de influencia; comunidad indígena

\section{Formative Process Representation of an Ethnic Education Institution through the Technique of Graphic and Descriptive Model Using Influence and Forrester's Diagrams}

\begin{abstract}
In this work a training process implemented in an ethnic education institution in an indigenous community in marginal conditions is described. The study follows a qualitative and quantitative methodology to develop the descriptive model. Computer graphics is employed to build influence diagrams and Forrester's diagrams. These diagrams allow graphically capturing the essence of this complex system and help to understand these relationships between variables of the training process. System dynamics makes visible the influence of independent variables that affect the educational process such as geographical location, date of teacher recruitment and transportation of children to school.
\end{abstract}




\section{INTRODUCCIÓN}

Conocer realidades es una actividad a partir del cual el hombre adquiere una certeza de lo que ella es (Abello, 2010) y la puede representar a través de símbolos y objetos con los cuales media esa verdad que va construyendo. Una forma de lograr este conocimiento del mundo es a través de modelos, y acorde a Izquierdo, (2008) teniendo claro el concepto de modelos, es la mejor forma de conocer la realidad. Lo que se pretende con esta representación no responde a la noción de construir un modelo que se entienda como una "matematización de la realidad" formulación de Israel citado por Armatte (2006), sino aprovechar el uso de la dinámica de Sistemas para construir el modelo descriptivo y gráfico a través de los diagramas de influencia o causales y de Forrester. Se cuantifican algunos aspectos de este fenómeno social apoyados en la estadística descriptiva, lo que permite construir más conocimiento agrupado y categorizado sobre el tema, y luego se pasa al modelo gráfico para el que se construyen diagramas causales o de influencia y diagramas de Forrester o de flujos con el uso de un programa informático que posee prestaciones diseñadas para la construcción de modelos de simulación basados en los conceptos de la dinámica de sistemas (Capelo y Días, 2009), (Gómez et all., 2015) y de pensamiento sistémico (Andrade, 2001).

Armatte (2006) ilustra cinco significaciones de modelo, como referente o prototipo a reproducir, como maqueta de un dispositivo real, como tipo ideal extraído de una población homogénea, como icono o dispositivo mecánico representando una idea abstracta, o el formalismo lógico-matemático que representa un sistema, en las que en las dos primeras significaciones, hay un prototipo, mientras que en las tres últimas hay un tipo, una abstracción de la realidad. Diferencias imperceptibles que enmarcan la forma como se construye conocimiento a partir de ellos. Nuestro interés está en la significación de tipo y se retoma el pensamiento de Izquierdo en el sentido que los modelos se construyen mediante procesos de abstracción con el objetivo de facilitar la comprensión de ciertos aspectos de un determinado sistema real. Se pretende hacer uso del profundo valor explicativo que tiene la descripción y la grafía del modelo, que teniendo en cuenta las apuestas de Bordieu señalado por Romero (2010), explica la acción que existe en la producción de este conocimiento social desde una práctica científica. El interés es ilustrar este complejo sistema social para que sus actores comprendan las relaciones y el impacto de sus dinámicas dentro de él, y se puedan tomar decisiones que modifiquen de manera positiva el sistema y también sirva como aporte para propiciar que otros actores sociales posean una nueva cosmovisión de un proceso formativo en una comunidad indígena en condiciones de marginalidad.

Los criterios que se tienen para usar los diagramas de Influencia y de Forrester como modelo gráfico están soportados en la concepción de que ellos son una forma de representación esquemática de la realidad que ayuda a visibilizar e ilustrar el complejo sistema real de forma que pueda ser comprensible para los actores sociales. El diagrama de Influencia es un diagrama que recoge los elementos claves del sistema y las relaciones entre ellos, (Aracill, 1995), que supera las representaciones que usa la Teoría General de Sistemas (Bertalanffy, 1976), porque posibilita ver como los eventos y sus influencias reproducen de manera activa la estructura básica del sistema históricamente, lo que se hace de manera gráfica usando flechas, signos y variables que representan eventos, lo que resulta ser fácil de identificar y comprender. Esto ayuda a construir el diagrama de Forrester que también es una representación gráfica de sistemas dinámicos y que modela cualitativamente las relaciones entre las partes mediante símbolos que corresponden a una interpretación dinámica del sistema. (Jiménez et all, 2002).

En la literatura científica hay documentación sobre el modelado de sistemas usando diagramas de influencia y de Forrester. En el Modelado de sistemas complejos mediante simulación basada en agentes y mediante dinámica de sistemas (2008), Izquierdo, Galán, Santos y del Olmo, realizan una comparación de la simulación basada en agentes y de la dinámica de sistemas como metodologías de modelado científico de sistemas complejos. Ceballos, Uribe, y Sánchez, (2013) en Modelo de Dinámica de Sistemas para la Predicción del Comportamiento del Mercado Porcícola, presentan la construcción de un modelo basado en dinámica de sistemas para describir el comportamiento del mercado porcícola, con el objetivo de anticiparse a los posibles acontecimientos, crear escenarios supuestos y determinar si es viable o no invertir en el corto a mediano plazo en este mercado. Los resultados de ese modelo muestran una gran dependencia del costo de la materia prima sobre las ganancias y establece el escenario de cría como el más rentable. Gómez Andrade y Vásquez, (2015), en Lineamientos Metodológicos para construir Ambientes de Aprendizaje en Sistemas Productivos Agropecuarios soportados en Dinámica de Sistemas, presentan una propuesta metodológica que orienta, facilita y motiva la construcción de ambientes de aprendizaje para la formación y entrenamiento sobre la toma de decisiones, la administración y la investigación de procesos agropecuarios soportado en la dinámica de sistemas. Todos ellos pretenden penetrar en la complejidad de los sistemas reales que modelan y las formas y maneras como se modifican sus comportamientos, a pesar de la complejidad dinámica de estos fenómenos sociales. Este trabajo que privilegia la explicación de la realidad a través de la descripción de ella, la ilustración y visibilización de las variables que están presentes en el proceso formativo, así como las relaciones que se configuran, puede ser útil como un escenario en el tiempo 
que identifique de manera estructural las razones que influencian su comportamiento y servir a futuro como base para parametrizar y simular desde la dinámica de sistemas los aspectos que están presentes en el proceso formativo.

La Institución etnoeducativa rural Laachon sobre la que se realiza el estudio, se encuentra ubicada en Manzana, al interior de una comunidad indígena asentada en La Guajira, Colombia, que habita en condiciones de marginalidad, las que se han agudizado en la actualidad por las condiciones ambientales de pocas lluvias (Ideam, 2015), en una zona semidesértica ubicada en una península que sobresale en el mar, al norte del país. La escuela es de modalidad de Etnoeducación, que promueve que haya entre los estudiantes wayuu un bilingüismo equilibrado en las dos lenguas, wayuu y español, y que no aprenda una en detrimento de la otra (González, 2015).

El análisis se consolida a partir de la caracterización de los estudiantes y docentes que conforman la comunidad educativa, teniendo como elementos básicos: acercamiento a las tecnologías de estudiantes y docentes, formación de los docentes, experiencia, grados a cargo, uso en el aula de clase de las tecnologías y una valoración inicial de la forma como realizan el acto de aula. Se desarrolló durante tres (3) meses, con jornadas diarias, y se usaron dos técnicas para recolectar información a tabular: entrevista a los docentes y estudiantes; los primeros, respondían a preguntas sobre la actividad laboral. La otra técnica, fue la observación directa del acto de aula, para la que se utilizó un instrumento estructurado en el que se identificaron prácticas de enseñanza. Para ambos casos, se realizó sobre el mismo universo de docentes. Los niños fueron entrevistados usando un formato de preguntas estructurado cuyo objetivo respondía a identificar si conocían tecnologías y si las sabían usar.

Para las entrevistas a docentes, se usaron cuestionarios con respuesta cerrada, y para la observación de la clase se utilizó un cuestionario de preguntas estructurado que atiende aspectos que se pueden encontrar durante el desarrollo de una clase y registrados de manera inmediata durante el desarrollo del acto de aula. Este proceso de observación y de entrevista estuvo a cargo de estudiantes de Ingeniería de Sistemas de la Universidad de La Guajira, orientados por la docente de la asignatura de Modelos, - asignatura del currículo del Programa de Ingeniería de Sistemas, en la que los estudiantes identifican técnicas y metodologías para representar y modelar cualquier sistema a partir de una realidad-, con el propósito de reconocer el complejo ecosistema social (Diez, 2001) (Morín E, Sánchez, A, 1981), que se configura en esa comunidad educativa. La presencia de estos estudiantes en el proyecto también incorpora sentido desde la ingeniería social para desarrollar proyectos de Investigación y de proyección social, en la que su interacción con la comunidad se gesta desde una mirada académica y no desde el asistencialismo. Se pretende con esto que el estudiante afine el primer nivel del conocimiento científico que corresponde a la observación y el segundo que corresponde a la descripción (Tamayo, 2004).

Para los autores se entiende el proceso formativo como un proceso en el cual un grupo de personas a partir de contenidos, habilidades y aptitudes se congregan o son congregados para aprender (Bolívar, 2003). A partir de eso se reconocen los actores de ese proceso formativo, que es un sistema, como: los que forman, en este caso los docentes, los formados, los estudiantes, los recursos, y el entorno o contexto donde se desarrolla la formación, y las relaciones que se dan entre estos elementos.

\section{METODOLOGÍA}

La metodología que se usa para el desarrollo de este reconocimiento, es de corte cuali-cuantitativa. Desde lo cualitativo se aborda el estudio de caso, cómo una herramienta de investigación, cuya mayor fuerza radica en que a través de ella se mide y registra la conducta de las personas involucradas en el fenómeno estudiado, (Martínez, 2006) en este caso, de los docentes participantes en el proceso formativo en la Institución etnoeducativa. El método cuantitativo, la tabulación de datos estadísticos, se usa para obtener información verbal a través de encuestas por cuestionarios, (ibídem) y se aplica a estudiantes y docentes de la misma Institución. Con esto se elabora el modelo descriptivo, y se usa la computación gráfica para construir los diagramas causales o de influencia y el diagrama de Forrester. Esta investigación está inmersa dentro de un Proyecto de investigación, innovación y desarrollo, denominado "Incorporación de las tecnologías móviles para potenciar los estilos de aprendizajes en la comunidad educativa del área de influencia de la Universidad de La Guajira: caso específico: estudiantes indígenas Wayuu" el que se estructura por fases formativas, a partir de metodologías cuantitativas y cualitativa, desde la Investigaciónacción-participación, (Ander-Egg, 2003) las que en su fase inicial identifican las condiciones en que se encuentran, y las necesidades básicas de las comunidades educativas indígenas de la etnia Wayuu.

Para la recolección de la información de la caracterización de los docentes se utilizaron técnicas cualitativas y cuantitativas (López, N., Sandoval, I., 2006.). Se tomó como objeto de estudio la población total de docentes que conforman la institución etnoeducativa rural Laachon Mayapo sede Manzana. La técnica 
utilizada fue la entrevista que tenía un encabezado en la que el docente indicaba su nombre completo, formación como docente, cursos en tic, institución donde los realizo y el instrumento constaba de 12 preguntas, las que eran de respuesta cerrada, descriptiva, con varias opciones y que debían explicarse. ¿Conoce usted un computador?, ¿Ha tenido contacto con un computador? ¿Cómo ha sido ese contacto y para qué? ¿Con qué frecuencia lo usa? ¿Qué herramienta del computador maneja?, ¿Que es lo que más sabe hacer en el computador? ¿Que partes forman o pertenecen a un computador? ¿Los programas que tiene el computador en su interior, como se denominan? ¿Para que el computador realice acciones, que se usa? ¿El computador en el aula de clases es? ¿Qué usos en el aula de clases se le puede dar al computador? ¿Qué herramienta prefiere usar como medio para educar a sus estudiantes?

Para la observación de la clase, se utilizó un formato de preguntas estructurado (10 preguntas), sobre aspectos inherentes al desarrollo de una clase, las que fueron observadas y registradas por estudiantes universitarios) ¿Cómo inicia la clase? ¿Cómo suele moverse durante la clase? ¿Cómo usa las manos? ¿Cómo usa la voz? ¿Cómo usa la mirada? ¿Qué hace para mantener el nivel de atención durante la clase? ¿Qué hace para pasar del final de un tema al inicio del siguiente? ¿Qué hace para mantener la disciplina en clase? ¿Qué hace para motivar la participación de los alumnos? ¿Cómo enfatiza los puntos importantes? ¿Qué hace para asegurarse de que la explicación se ha entendido? ¿Qué hace durante el descanso? ¿Cómo utiliza las preguntas a los alumnos durante la clase? ¿Cómo finaliza la clase?

Para la recolección de la información sobre los estudiantes se tuvo en cuenta a los niños de 5to grado, (30 estudiantes) y se construyó un instrumento que se imprimió y con el que los estudiantes universitarios entrevistaban a cada niño, y registraban sus respuestas en el formato de manera escrita. A pesar de que las preguntas dispuestas en el instrumento impreso tenían gráficas, se necesitaba la ayuda y apoyo de los docentes bilingües del 5to grado, por cuanto el formato estaba en idioma español, y la lengua nativa de los niños, es el Wayunaiki, aunque en su gran mayoría comprendían el español muy bien.

¿Cuál de estos dibujos es un Computador? ¿Has usado alguna vez un computador? ¿Logras imaginarte lo que es un computador? ¿Sabes para qué sirve un computador? ¿Qué te gustaría hacer con un computador? ¿Qué has hecho en un computador? ¿Qué ha sido lo que más te ha gustado de un computador? ¿Te gustaría tener un computador en tu salón de clase? ¿Por qué? ¿Tienes y usas un celular? ¿Para qué lo usas? ¿Qué tipo de celular es? ¿En tu casa hay quien tenga un celular? ¿Quién? ¿Lo has usado tú? ¿Ves la Televisión en casa? ¿En la escuela? ¿Qué programas observas? ¿Por qué los ves? ¿Escuchas la Radio en casa? ¿En la escuela? ¿Qué programas escuchas? ¿Te gusta leer? ¿Te gusta la cartilla de la escuela? ¿Qué libro te gusta leer? ¿Te gusta dibujar? Si _ No _. ¿Por qué?

El interés del equipo investigador en identificar el nivel de uso de las tic en el proceso formativo, radica en el macroproyecto de investigación que está asociado a este, se sustenta desde la postura que las tic producen cambios en el proceso de construcción de conocimiento de manera que lo favorecen (Ricoy, Feliz y Sevillano, 2010). Se asume que estas generan cambios significativos no solo en el aprendizaje sino en la motivación e interés, en el rendimiento académico y en el desarrollo de nuevas competencias. Aunque esto requiere un cambio organizativo y una reelaboración de las propuestas didácticas tradicionales, adaptándolas a un nuevo modelo pedagógico específico (Expósito y Manzano, 2012).

\section{RESULTADOS Y DISCUSIÓN}

\section{Modelo descriptivo del proceso formativo}

Se utiliza la técnica del modelo descriptivo (Aguilar, 2004) para presentar el contexto en el que está inmersa la Institución etnoeducativa.

La Institución Rural Laachon Mayapo Sede Manzana es una sede etnoeducativa que cuenta con un equipo de docentes constituidos por: 2 docentes de planta, nombrados por el Ministerio de Educación Nacional de Colombia, - MEN - y 10 docentes contratados por una Agencia Operadora de educación para comunidades indígenas, que se encarga anualmente de administrar la atención educativa para la construcción e implementación del Sistema Educativo Propio, que responde a la puesta en marcha de un sistema educativo que este contextualizado a su condición indígena, lengua y cosmovisión. Para el año 2015 fue contratada Wayuu Araurayu (Gobernación de La Guajira, 2015) para administrar esta institución etnoeducativa. Este proceso de contratación se da entre el gobierno departamental y los operadores educativos, por convocatoria pública abierta los primeros días del año. Cada año los docentes de planta inician sus labores en el tiempo establecido, la segunda semana de enero, mientras que por su parte, cada año los docentes contratados deben esperar hasta firmar un contrato para iniciar por completo sus clases. Los períodos de tiempo que se dan entre el inicio del año y el tiempo que se toman estos procesos jurídicoadministrativos para ponerse a tono y completar los requisitos, puede tomar alrededor de tres (3) meses. 
Los docentes que laboran en la Institución etnoeducativa orientan las actividades de enseñanza-aprendizaje de cada grado escolar y su formación es de nivel técnico, tecnológico y superior, lo que influye en la planeación de la clase, la atención de los niños y su forma de enseñar. La institución etnoeducativa, no cuenta con aula de informática y no usan computador, ni ninguna tecnología en el aula como complemento a la acción formativa. Los estudiantes manifiestan no tener acceso a las tic. La tecnología que más conocen y usan los niños, es la radio y la televisión, y los que menos la computadora personal (en adelante pc) y el teléfono celular. Estas tecnologías las conocen porque la usan sus padres y hermanos mayores. Los niños manifiestan que escuchan radio porque sus padres lo usan para escuchar partidos de futbol, música 0 noticias. Igual ocurre con la televisión en la que también ven programas para niños, que en su mayoría se pueden identificar historietas animadas con un alto contenido de violencia.

En esta institución etnoeducativa, los estudiantes procuran asistir a la escuela, pero muchos de ellos viven en lugares aledaños a donde queda ubicada la sede educativa. Estos lugares están entre 1.5 y 3 kilómetros de distancia y su asistencia a la escuela no es permanente. Ellos llegan a la institución de forma intermitente, van a pie hasta la escuela, con altas temperaturas climáticas, por cuanto la vegetación es escasa, la que corresponde a zona semidesértica, que es lo que predomina en esta zona de la media Guajira. Dentro de lo que se contrata anualmente para desarrollar el servicio educativo, está el servicio de transporte, que se formaliza unas semanas después que han sido contratados los Operadores de Educación y los docentes, lo que puede tomar aproximadamente unos dos o tres semanas y de esa forma se cuenta con este servicio casi hasta el cuarto mes del año.

En la figura 1 se presenta la ubicación de la institución etnoeducativa de Manzana y la de algunos de los niños que estudian en ella y no habitan en el mismo lugar donde está localizada la sede de la institución. El círculo amarillo más grande corresponde a la ubicación geográfica de la Institución educativa y los círculos amarillos más pequeños representan la ubicación donde habitan algunos niños que estudian allí.

La Institución Etnoeducativa Laachon Mayapo sede Manzana, tiene grados desde preescolar hasta quinto de primaria, y hay dos cursos por cada grado, a excepción de preescolar, en el que solo hay uno. En sus documentos institucionales reportan 246 estudiantes matriculados, con una asistencia regular de 156 estudiantes. Los demás son intermitentes o desertan. Los docentes utilizan el marcador, el tablero y el libro de texto para orientar la clase. Las formas de evidenciar que se termina un tema o se continúa en el mismo, o preguntar o sancionar por una actividad no realizada o aprobar por una que si se realiza, es a través de frases, las que se hacen en un tono de voz diferente. Si es aprobado, la voz es agradable y si es lo contrario, el tono de voz es alto y a modo de regaño. La mayoría de los docentes se pasean por el salón, y se acercan a los estudiantes a observar si están desarrollando las actividades. Con una excepción, una docente que siempre está en el pupitre del docente adelante, todos se mueven por el salón de clases. Durante los primeros meses del año, los docentes de planta realizan la labor escolar y la orientación de la mayoría de los cursos, teniendo en cuenta la asistencia de los niños a la escuela. Mientras se contratan a los docentes anuales, se dedican a repasar los temas enseñados el año anterior. Acorde a lo que ellos manifiestan sobre si mismos en la entrevista que se les hace, la mayoría se encuentran en proceso formativo en la Universidad de La Guajira en licenciatura en Etnoeduación. Algunos manifiestan que aunque recibieron capacitación en tic, no la recuerdan ni la han usado nunca en el aula de clases. Desde el modelo gráfico (Macleod, 2000), se pretende conectar la descripción y las gráficas que se generan, para explicar, comprender y representar aspectos de esta realidad a través de las tablas y diagramas, que la resumen y documentan de manera estadística y visual. En las tablas 1 y 2 se presenta información categorizada de estudiantes y docentes.

Tabla 1. Información sobre formación, experiencia docente, formación en tic, contacto en tic y uso de tic en el aula, sobre los docentes que laboran en la Institución Etnoeducativa Laachon sede Manzana.

\begin{tabular}{|l|c|c|c|c|c|c|c|}
\hline \multicolumn{2}{|c|}{$\begin{array}{c}\text { Formación Docentes } \\
\text { Manzana }\end{array}$} & \multicolumn{2}{c|}{ Experiencia Docente } & \multicolumn{2}{c|}{ Formación en TIC } & \multicolumn{2}{c|}{$\begin{array}{c}\text { Uso de TIC en } \\
\text { el Aula }\end{array}$} \\
\hline $\begin{array}{l}\text { Universitario } \\
\text { (cursando 7, } \\
\text { terminado 1) }\end{array}$ & 8 & Entre1-4 años & 5 & $\begin{array}{c}\text { Servicio Nacional de } \\
\text { Aprendizaje (SENA, } \\
\text { educación no formal) }\end{array}$ & 6 & SI & 0 \\
\hline Normalista & 2 & Entre 5-9 años & 4 & Diplomados & 5 & No & 11 \\
\hline Técnico & 1 & $\begin{array}{c}\text { Entre 10-22 } \\
\text { años }\end{array}$ & 2 & & & & \\
\hline Total & 11 & Total & 11 & Total & 11 & Total & 11 \\
\hline
\end{tabular}




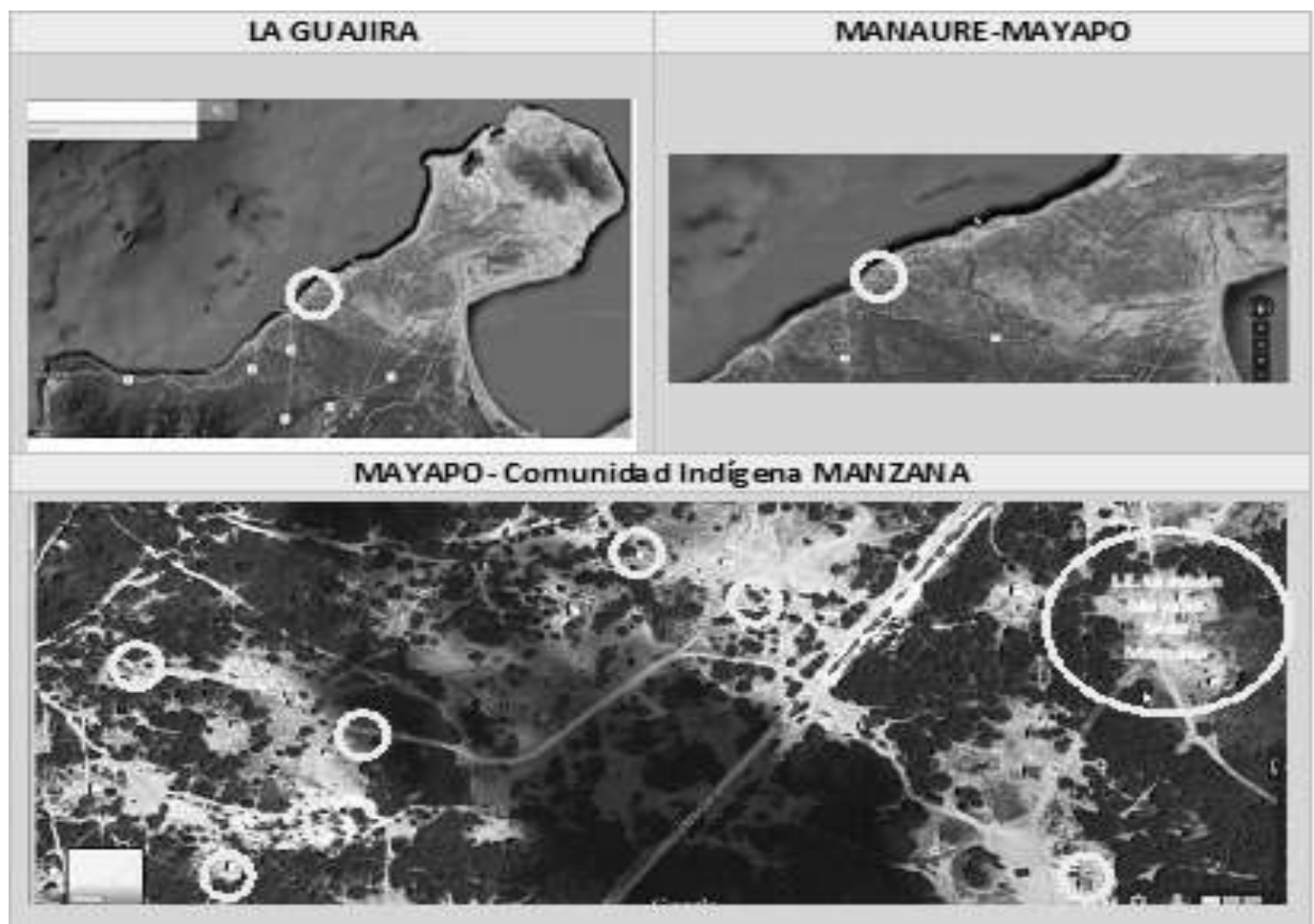

Fig. 1. Imagen tomada de Google earth modificada por los autores, que muestra la ubicación de la I.E y donde habitan los niños.

Tabla 2. Información sobre conocimiento y uso de pc, uso de teléfono celular, radio y tv por los niños que estudian en la Institución Etnoeducativa Laachon Mayapo, sede Manzana.

\begin{tabular}{|l|l|l|l|l|l|l|l|}
\hline $\begin{array}{c}\text { Conocimiento y Uso De } \\
\text { PC }\end{array}$ & \multicolumn{3}{c|}{ Uso Celular } & \multicolumn{3}{c|}{ Uso Radio } & \multicolumn{3}{c|}{ Uso TV } \\
\hline $\mathrm{Si}$ & 7 & $\mathrm{Si}$ & 2 & $\mathrm{Si}$ & 103 & $\mathrm{Si}$ & 97 \\
\hline $\mathrm{No}$ & 149 & No & 154 & No & 53 & No & 59 \\
\hline Total & 156 & Total & 156 & Total & 156 & Total & 156 \\
\hline
\end{tabular}

Usando el modelo descriptivo se analizan e identifican las variables y su tipo, independientes, aquellas que son susceptibles de ser modificadas desde el exterior del sistema y las dependientes cuyo comportamiento está completamente determinado por la estructura del sistema, (Díaz, 2010). Estas variables servirán para construir un modelo gráfico usando diagramas de Influencia y de Forrester sobre el proceso formativo. Estas variables por clasificación se presentaran como variables asociadas a los docentes Tabla 3 y variables asociadas a los estudiantes Tabla 4.

\section{Variables asociadas a los Docentes}

En la tabla 3, se presentan cada una de las variables asociada con los docentes.

Tabla 3. Variables identificadas asociadas a los docentes.

\begin{tabular}{|c|c|c|c|}
\hline Variables/docentes & Definición & Tipo & justificación \\
\hline Docentes en la escuela & $\begin{array}{l}\text { Corresponde a todos los docentes de } \\
\text { planta y contratados que integran la } \\
\text { Institución Etnoeducativa }\end{array}$ & dependiente & $\begin{array}{ll}\text { Es influenciada por la } \\
\text { variable } & \text { docentes } \\
\text { contratados } & \end{array}$ \\
\hline Docentes de planta & $\begin{array}{l}\text { Son docentes que de manera permanente } \\
\text { pertenecen a la Institución Etnoeducativa }\end{array}$ & independiente & $\begin{array}{l}\text { Depende de cupos que } \\
\text { asignen a la Institución } \\
\text { educativa y depende de } \\
\text { recursos presupuestales } \\
\text { que tenga el municipio para } \\
\text { docentes permanentes. }\end{array}$ \\
\hline Proporción de docentes de planta & $\begin{array}{l}\text { Corresponde a la tasa de docentes de } \\
\text { planta con relación al total de docentes } \\
\text { que laboran en la Institución } \\
\text { Etnoeducativa. }\end{array}$ & dependiente & $\begin{array}{l}\text { Depende de la cantidad de } \\
\text { docentes de planta y de } \\
\text { docentes contratados }\end{array}$ \\
\hline
\end{tabular}


Tabla 3. Continuación.

\begin{tabular}{|c|c|c|c|}
\hline Docentes contratados & $\begin{array}{l}\text { Corresponde al número total de docentes } \\
\text { contratados en la la Institución } \\
\text { Etnoeducativa Laachon Mayapo sede } \\
\text { Manzana. }\end{array}$ & dependiente & $\begin{array}{l}\text { La cantidad de estudiantes } \\
\text { en la escuela impacta } \\
\text { docentes que se contraten }\end{array}$ \\
\hline Proporción docentes contratados & $\begin{array}{l}\text { Tasa de docentes que son contratados } \\
\text { anualmente en Institución Etnoeducativa. }\end{array}$ & dependiente & $\begin{array}{l}\text { Depende de la cantidad de } \\
\text { docentes contratados }\end{array}$ \\
\hline $\begin{array}{l}\text { Contratos por año Operador } \\
\text { Educativo }\end{array}$ & $\begin{array}{l}\text { Son los contratos que realiza cada año el } \\
\text { Operador Educativo para poner en marcha } \\
\text { el servicio en la Institución etnoeducativa } \\
\text { Laachon. }\end{array}$ & independiente & $\begin{array}{l}\text { Depende de los recursos } \\
\text { asignados por el Gobierno } \\
\text { departamental para el } \\
\text { proceso de operación del } \\
\text { servicio educativo }\end{array}$ \\
\hline $\begin{array}{l}\text { Docentes finalizan contrato cada } \\
\text { año }\end{array}$ & $\begin{array}{l}\text { Son los docentes que finalizan el contrato } \\
\text { al terminar el año escolar en la Institución } \\
\text { Etnoeducativa }\end{array}$ & dependiente & $\begin{array}{l}\text { Depende de los docentes } \\
\text { contratados }\end{array}$ \\
\hline Formación de los docentes & $\begin{array}{l}\text { Corresponde a toda la capacitación formal } \\
\text { y no formal en cursos, que puede servir } \\
\text { para su rol como docentes, con que } \\
\text { cuentan los docentes de planta y } \\
\text { contratados de la Institución } \\
\text { Etnoeducativa }\end{array}$ & dependiente & $\begin{array}{l}\text { La necesidad de hacer } \\
\text { parte del proceso formativo, } \\
\text { influencia esta formación, o } \\
\text { como necesidad de cumplir } \\
\text { su rol formador }\end{array}$ \\
\hline $\begin{array}{l}\text { Proporción de formación de los } \\
\text { docentes }\end{array}$ & $\begin{array}{l}\text { Es la tasa que representa el porcentaje } \\
\text { asociado a los cursos realizados por los } \\
\text { docentes. }\end{array}$ & dependiente & $\begin{array}{l}\text { Depende de la formación } \\
\text { que tengan los docentes }\end{array}$ \\
\hline Formación para ser docentes & $\begin{array}{l}\text { Corresponde a la preparación profesional, } \\
\text { técnica o cursos realizados para ejercer la } \\
\text { docencia. }\end{array}$ & dependiente & $\begin{array}{l}\text { La necesidad de hacer } \\
\text { parte del proceso formativo } \\
\text { de manera específica, } \\
\text { influencia esta formación, o } \\
\text { como necesidad de cumplir } \\
\text { su rol formador }\end{array}$ \\
\hline Otra formación & $\begin{array}{l}\text { Son cursos de cualquier nivel que no } \\
\text { corresponden a la formación para ejercer } \\
\text { la docencia }\end{array}$ & independiente & $\begin{array}{l}\text { Puede ser realizada antes } \\
\text { de ingresar al proceso } \\
\text { formativo. }\end{array}$ \\
\hline Calidad en la forma de enseñanza & $\begin{array}{l}\text { Es la forma como se desarrolla el proceso } \\
\text { de enseñanza-aprendizaje en los } \\
\text { docentes y estudiantes de la Institución } \\
\text { Etnoeducativa }\end{array}$ & dependiente & $\begin{array}{l}\text { Es influenciada por la } \\
\text { formación del docente, y } \\
\text { por la experiencia como } \\
\text { docente }\end{array}$ \\
\hline Formación en TIC & $\begin{array}{l}\text { Son todas las horas dedicadas a estudiar } \\
\text { sobre las tecnologías de la información y } \\
\text { comunicación por los docentes con } \\
\text { sentido pedagógico. }\end{array}$ & independiente & $\begin{array}{l}\text { El docente no ve esto como } \\
\text { una necesidad, y puede } \\
\text { tenerla o no tenerla }\end{array}$ \\
\hline Educación No formal & $\begin{array}{l}\text { Corresponde a cursos cortos sobre } \\
\text { tecnologías de la información y } \\
\text { comunicación, tales como: cursos en } \\
\text { Sena, diplomados, cursos técnicos. }\end{array}$ & independiente & $\begin{array}{l}\text { El docente no ve esto como } \\
\text { una necesidad, porque la } \\
\text { forma como es contratado } \\
\text { pesa su condición de nativo } \\
\text { y el hecho de estar en la } \\
\text { escuela }\end{array}$ \\
\hline Experiencia como docente & $\begin{array}{l}\text { Son los años y cursos sobre el cual él ha } \\
\text { estado docente ejerciendo }\end{array}$ & dependiente & $\begin{array}{l}\text { Es influenciado por los } \\
\text { grados asignados durante } \\
\text { cada año }\end{array}$ \\
\hline Grados asignados & $\begin{array}{l}\text { Son los grados que van de } 0 \text { grado a } 5 \\
\text { grado de primaria que están en la } \\
\text { Institución Etnoeducativa, que se asignan } \\
\text { a los docentes }\end{array}$ & dependiente & $\begin{array}{l}\text { Depende de la cantidad de } \\
\text { estudiantes asignados }\end{array}$ \\
\hline Medios tecnológicos en la escuela & $\begin{array}{l}\text { Son cada uno de los medios tecnológicos } \\
\text { con los que cuenta la escuela Institución } \\
\text { Etnoeducativa }\end{array}$ & dependiente & $\begin{array}{l}\text { Es influenciado por los } \\
\text { recursos que tenga la } \\
\text { escuela }\end{array}$ \\
\hline Uso de TIC en el aula & $\begin{array}{l}\text { Es el uso de las tecnologías de la } \\
\text { información y la comunicación por parte } \\
\text { de los docentes en el aula para enseñar }\end{array}$ & dependiente & $\begin{array}{l}\text { Es influenciado por la } \\
\text { formación que tengan los } \\
\text { docentes para usar las tic } \\
\text { en el aula }\end{array}$ \\
\hline Proporción uso de tic en el aula & $\begin{array}{l}\text { Es la tasa de uso de tic en el aula que } \\
\text { hagan los docentes }\end{array}$ & dependiente & $\begin{array}{l}\text { Es influenciado por el uso } \\
\text { de las tic en el aula }\end{array}$ \\
\hline Preparación de la clase & $\begin{array}{l}\text { Corresponde a tiempo y sentido del } \\
\text { docente previo para organizar la clase }\end{array}$ & dependiente & $\begin{array}{l}\text { Es influenciado por los } \\
\text { docentes contratados y la } \\
\text { formación para ser docente }\end{array}$ \\
\hline Volumen de voz del docente & $\begin{array}{l}\text { Nivel con el cual el docente usa su voz } \\
\text { durante la sesión de clases }\end{array}$ & dependiente & $\begin{array}{l}\text { Es influenciado por la } \\
\text { atención de los estudiantes } \\
\text { en la clase }\end{array}$ \\
\hline
\end{tabular}




\section{Variables asociadas a los Estudiantes}

En la tabla 4, se presentan cada una de las variables asociada con los estudiantes de la institución:

Tabla 4. Tabla de variables asociadas a los estudiantes.

\begin{tabular}{|c|c|c|c|}
\hline Variables/Estudiantes & Definición & Tipo & Justificación \\
\hline $\begin{array}{l}\text { Estudiantes en la } \\
\text { escuela }\end{array}$ & $\begin{array}{l}\text { Corresponde al total de los estudiantes que } \\
\text { están matriculados y forman parte de cada } \\
\text { uno de los grados con los que cuenta la } \\
\text { Institución Etnoeducativa y que permanecen } \\
\text { a la escuela. }\end{array}$ & dependiente & $\begin{array}{l}\text { Es influenciado por los } \\
\text { grados con que cuenta la } \\
\text { escuela, los docentes de } \\
\text { la escuela, y los } \\
\text { estudiantes que asisten }\end{array}$ \\
\hline Estudiantes asistentes & $\begin{array}{l}\text { Son todos los estudiantes que asisten de } \\
\text { manera regular a la Institución } \\
\text { Etnoeducativa }\end{array}$ & independiente & $\begin{array}{l}\text { Estudiante que quiere } \\
\text { cumplimentar el proceso } \\
\text { formativo }\end{array}$ \\
\hline $\begin{array}{l}\text { Proporción de } \\
\text { estudiantes asistentes }\end{array}$ & $\begin{array}{l}\text { Es la tasa de los estudiantes que asisten a } \\
\text { la Institución Etnoeducativa, respecto a la } \\
\text { población total de estudiantes }\end{array}$ & dependiente & $\begin{array}{l}\text { Depende de los } \\
\text { estudiantes asistentes }\end{array}$ \\
\hline $\begin{array}{l}\text { Estudiantes } \\
\text { intermitentes }\end{array}$ & $\begin{array}{l}\text { Son todos los estudiantes que asisten de } \\
\text { manera irregular la Institución Etnoeducativa }\end{array}$ & dependiente & $\begin{array}{l}\text { Es el estudiante que no } \\
\text { asiste a la escuela de } \\
\text { manera regular }\end{array}$ \\
\hline $\begin{array}{l}\text { Proporción estudiantes } \\
\text { intermitentes }\end{array}$ & $\begin{array}{l}\text { Corresponde a la tasa de porcentaje de } \\
\text { estudiantes que asisten de manera irregular } \\
\text { la Institución Etnoeducativa }\end{array}$ & dependiente & $\begin{array}{l}\text { Depende de los } \\
\text { estudiantes asistentes }\end{array}$ \\
\hline Estudiantes desertores & $\begin{array}{l}\text { Corresponde a la cantidad de estudiantes } \\
\text { que abandonan la Institución Etnoeducativa } \\
\text { y no asisten más a la escuela }\end{array}$ & dependiente & $\begin{array}{l}\text { influenciado por la } \\
\text { distancia de la escuela y la } \\
\text { carencia de transporte } \\
\text { escolar; pueden volverse } \\
\text { estudiantes desertores }\end{array}$ \\
\hline $\begin{array}{l}\text { Proporción estudiantes } \\
\text { desertores }\end{array}$ & $\begin{array}{l}\text { Corresponde a la tasa de porcentaje de } \\
\text { estudiantes que desertan de la Institución } \\
\text { Etnoeducativa }\end{array}$ & dependiente & $\begin{array}{l}\text { Depende de los } \\
\text { estudiantes desertores }\end{array}$ \\
\hline $\begin{array}{l}\text { Atención de los } \\
\text { estudiantes en la clase }\end{array}$ & $\begin{array}{l}\text { Cantidad de atención que prestan los } \\
\text { estudiantes a los docentes en la clase }\end{array}$ & dependiente & $\begin{array}{l}\text { Es influenciado por la } \\
\text { preparación que hace el } \\
\text { docente de la clase y por } \\
\text { la calidad de la forma de la } \\
\text { enseñanza }\end{array}$ \\
\hline Transporte escolar & $\begin{array}{l}\text { Existencia de un vehículo que utilizan como } \\
\text { medio de transporte que lleva a los niños a } \\
\text { la escuela. }\end{array}$ & dependiente & $\begin{array}{l}\text { Es influenciado por los } \\
\text { contratos del Operador de } \\
\text { servicios educativos }\end{array}$ \\
\hline Distancia escuela & $\begin{array}{l}\text { Corresponde a un valor entre } 1.5 \text { a } 3 \\
\text { kilómetros de distancia de las comunidades } \\
\text { indígenas a la escuela en la Institución } \\
\text { Etnoeducativa }\end{array}$ & independiente & Ubicación geográfica \\
\hline
\end{tabular}

En la figura 2, se presenta el diagrama de influencias, en la que se observan a través de las variables y sus relaciones, las razones por las que el sistema se comporta, como lo hace. En la fig. 3 que corresponde al diagrama de Forrester, el cual fue realizado haciendo uso del software Vensim Ple, lo que se presenta es como se da el flujo de información o de material entre todas las variables identificadas que intervienen dentro del proceso formativo.

De acuerdo a los principios interpretativos de la dinámica de sistemas, Y se encuentra que la cantidad de docentes en la institución depende de la cantidad de docentes contratados y de los docentes de planta, y la cantidad de estudiantes en la Institución etnoeducativa es igual a los estudiantes que asisten más la sumatoria de los estudiantes que desertan y los estudiantes intermitentes, que están asociados a la distancia del lugar en que viven y la institución educativa. El desequilibrio entre la fecha de ingreso de los docentes contratados cada año y los de planta, genera atraso escolar cada año para cada curso, por el mecanismo que utilizan los segundos de repaso del año lectivo anterior. Para el proceso formativo, existen 3 calendarios: el calendario de inicio del año, segunda semana de enero, el calendario de contratación de los docentes, marzo y el calendario de contratación del transporte, 2 o 3 semanas después de la contratación de los docentes. El lugar donde habitan los niños es uno de los factores que influencia la asistencia 0 
deserción de la escuela, e influye de manera negativa la distancia de los niños a la escuela, porque no permite el acceso al aula de manera permanente, y se configura una geopedagogía, (Martínez B, 2000), en la que se reconoce que el acto de enseñar se restringe a pocos, por razones del acceso a la escuela. Esto se percibe como generador de desigualdad, exclusión (Jiménez, 2008) y desequilibrio social. Se hace necesario la implementación de un transporte que esté disponible desde el inicio de clases, para que los niños puedan usarlo y asistir a la institución educativa.

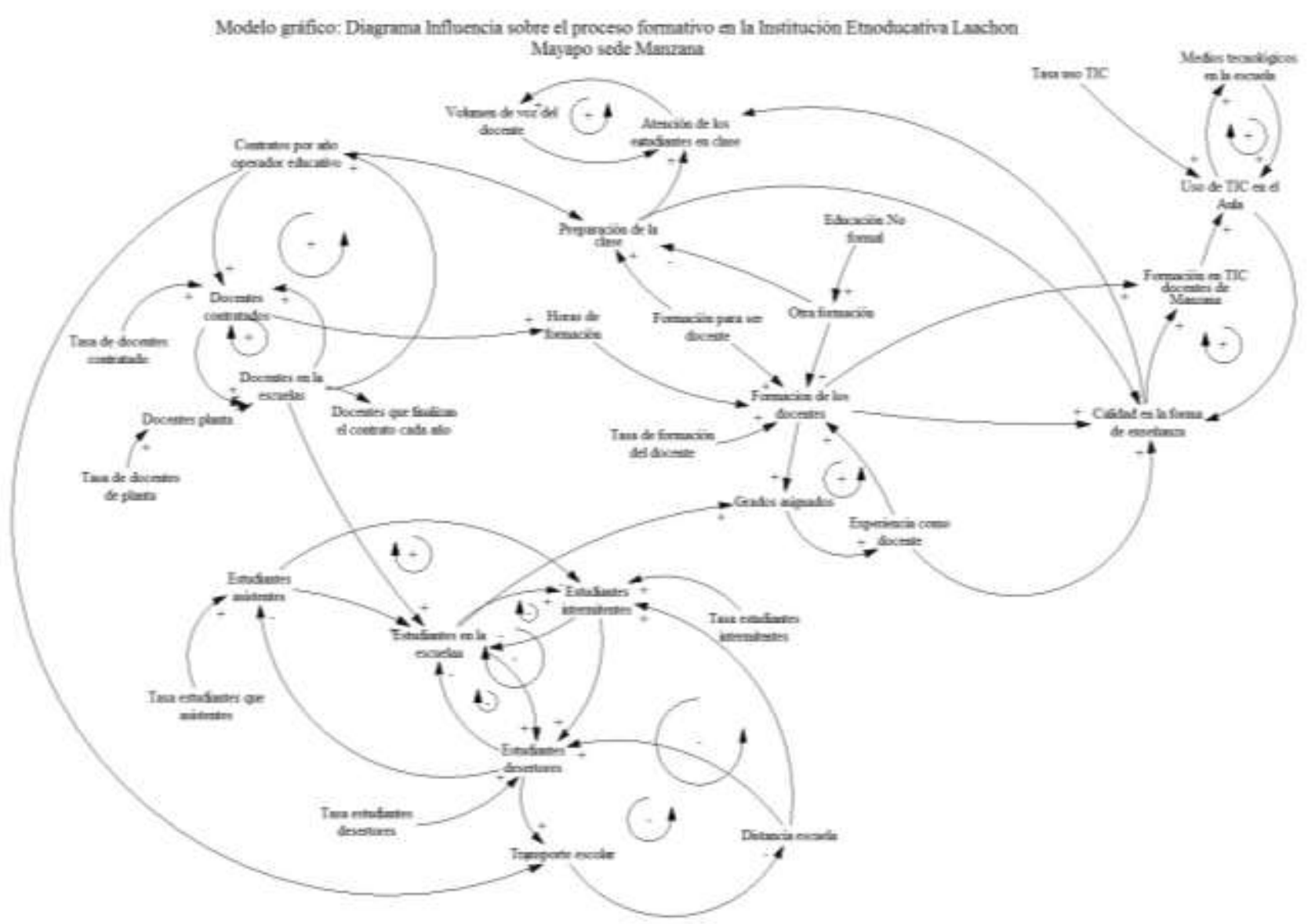

Fig. 2. Diagrama de influencia que soporta el modelo gráfico sobre el proceso formativo en la Institución Etnoeducativa Laachon sede Manzana.

Hay cursos que son afectados de manera directa por el proceso que demora en realizarse la contratación. Aquellos a los que están asignados los docentes que se contratan anualmente. La demora en el proceso de contratación del Operador para el servicio educativo de las Comunidades indígenas, se ha vuelto una costumbre conocida por los estudiantes, quienes tardan en llegar a la institución educativa. En algunos casos, los docentes tienen que ir a las casas a reclutar a los estudiantes.

La formación de los docentes impacta su forma de enseñar. Se puede relacionar la baja formación que tienen con las acciones que usan en el aula para orientar el proceso educativo. Los docentes en su gran mayoría no han terminado la carrera profesional como docente, sino que la están cursando, y es importante resaltar que el Operador de Servicios educativos no tiene en cuenta esta consideración al momento de contratarlo. Se considera que en esto impacta la condición de que los docentes sean todos nativos, indígenas wayuu de la localidad, que han laborado como docentes y tienen una básica formación en cualquier área, por encima de la educación de bachillerato.

Los docentes en el aula de clases para realizar la labor formativa siempre usan los mismos recursos, voz, tablero, libro y cuaderno, y de la misma forma; no se observan otras acciones didácticas para propiciar el aprendizaje. La mayoría de los docentes no han usado nunca un computador y se sienten intimidados por ello. Aunque los estudiantes nunca han usado un computador, frente a una gráfica en la que se presentan tres imágenes diferentes, identifican de manera inmediata al pc, lo que se reconoce como conocimiento tácito de los estudiantes, tal como lo identifican (Aarón, Castañeda, Ibarra, 2014). El uso de las tic en el aula, no solo está asociado al conocimiento que tengan los docentes sobre ellas, sino también a la 
ausencia/presencia de estos equipos informáticos en la escuela. Se considera que las condiciones de marginalidad en las que se encuentran los niños que habitan en la comunidad indígena de Manzana, no favorecen el buen desarrollo de su proceso formativo, y la forma como se desarrolla el proceso formativo en la Institución, no propicia que los niños puedan avanzar significativamente y trascender de esas condiciones de marginalidad.

\section{El Modelo gráfico}

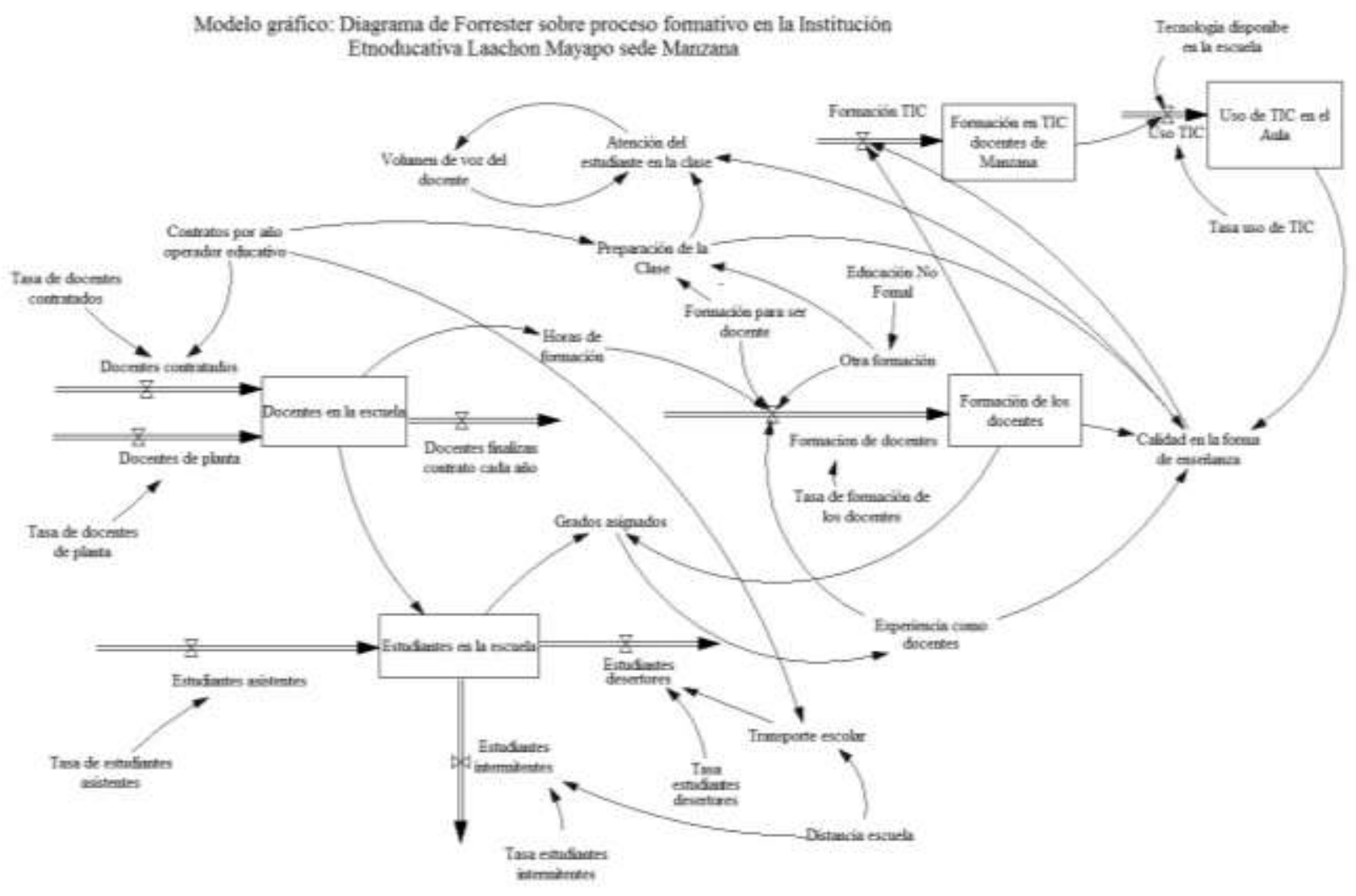

Fig. 3. Diagrama de forrester que soporta el modelo gráfico sobre el proceso formativo en la Institución Etnoeducativa Laachon sede Manzana.

\section{CONCLUSIONES}

A partir de lo observado e ilustrado, se puede concluir lo siguiente: 1) el modelo descriptivo y gráfico muestra que están presentes los elementos del proceso formativo, los estudiantes, los docentes, los recursos, y el contexto o entorno en el cual se desarrolla. 2) La identificación de las variables ayudó a la construcción de los modelos gráficos: influencia y de Forrester, y a reconocer la dinámica de estos sistemas reales. Se reconoce la dificultad para la identificación de variables que afectan de manera directa e indirecta procesos formativos. Son realidades complejas, se considera que las aquí identificadas pueden servir para el análisis de otros sistemas sociales con características similares a estas y pueden parámetros claves para la simulación de este proceso formativo en el tiempo. 3)Las condiciones administrativas para la contratación del servicio educativo, y el transporte a la escuela, influencia de manera directa el proceso formativo y genera exclusión que cada vez afecta más a esta comunidad indígena. Esta situación no es de la gobernabilidad de la Institución etnoeducativa. 4) En un ecosistema social se generan relaciones entre los miembros que lo componen, que presentan un alto nivel de complejidad. Esas relaciones son difíciles de regular o de controlar. Si esto fuera posible, el desarrollo del ecosistema social podría mejorar en beneficio de sus miembros. En este sistema educativo, se considera difícil de controlar las relaciones entre: estudiantes desertores, estudiantes intermitentes y transporte escolar. Así como formación, formación como docentes y calidad de la forma de enseñanza. El tiempo en el que se da la contratación de docentes, es la variable que está más fuera de control del ecosistema formativo.5) La caracterización de los docentes y los estudiantes, así como la observación y descripción del sistema a través de modelos descriptivos y gráficos, pueden ser herramientas útiles para la comprensión del sistema real. 6) Las representaciones visuales, en este caso, los diagramas de influencia y diagramas de Forrester, que utilizan unos símbolos gráficos que 
sirven para relacionar las razones y las secuencias de comportamiento de los diferentes eventos en forma diagramática, pueden ayudar a comprender mejor realidades complejas.

\section{AGRADECIMIENTOS}

A la Universidad de La Guajira. A Delfina Díaz, la rectora de La Institución Etnoeducativa Laachon Mayapo, a la docente Ena Vanegas, y todos los miembros de la Comunidad indígena, docentes y estudiantes de Manzana-Mayapo-Manaure.

\section{REFERENCIAS}

Aarón, M., Ibarra A y Castaneda O. The Management and Construction of Knowledge as an Innovation Strategy for Collaborative Learning Through the Use and Creation of Learning Communities and Networks, http://goo.gl/5m3FKf, ISSN: 1367-3270. International Journal of Knowledge Management, 38-49 (2014)

Aguilar G. F. Teoría de la decisión e incertidumbre: modelos normativos y descriptivos. Revista de Metodología de Ciencias Sociales, http://goo.gl/FGQoXW Empiria (8) Uned., 139-160 (2004)

Andrade H, Dyner I, Espinosa A, López H, Sotaquirá R.; Pensamiento Sistémico: Diversidad en búsqueda de Unidad. Ediciones Universidad Industrial de Santander (2001)

Andrade $\mathrm{H}$, Lince $\mathrm{E}$, Mercado A, Monsalve A. Evolución: herramienta software para modelado y simulación con Dinámica de Sistemas. Revista de Dinámica de Sistemas Vol. 5 Núm. 1. (2011)

Aracil, J, Gordillo F. Dinámica de sistemas. http://goo.gl/NXHylc. Isdefe, (1995)

Ander-Egg, E. Repensando la Investigación-Acción-Participativa. Grupo Editorial, 4ª edición, 29-42. Lumen Humanitas (2003)

Bertalanffy von L. Teoría General de los sistemas (2 $2^{\underline{a}}$ ed.) (en papel), s.l. Fondo de cultura económica de España, ISBN 9788437500539 (1976)

Bolívar, A. Didáctica y currículum. Retos, 15, 16. (2003)

Capelo, C. y Dias, J. F., A system dynamics-based simulation experiment for testing mental model and performance effects of using the balanced scorecard, http://goo.gl/Bom7Fb. System Dynamics Review, 25(1), 1-34 (2009)

Ceballos, Y. F., Uribe, M., y Sánchez, G. Modelo de Dinámica de Sistemas para la Predicción del Comportamiento del Mercado Porcícola. Inf. tecnol. [online]., vol.24, n.4 [citado 2016-01-22],pp.11712.http://dx.doi.org/10.4067/S0718-07642013000400013.Disponible en: <http://www.scielo.cl/scielo.php?script=sci_arttext\&pid=S0718-07642013000400013\&lng=es\&nrm=iso >. ISSN 0718-0764. (2013)

Díaz, M. Á. R., Merino, A. P., y Castellanos, R. S. M., Modelos de ecuaciones estructurales. Papeles del psicólogo, 31(1), 34-45. (2010)

Díez, J. Ecología Humana y Ecosistema social. Sociología y Medio Ambiente. ISBN 84-7433-228-1, pags. 17-32. (1982)

Dieterich, H, Nueva Guía para la Investigación científica. Impreso y hecho en México Decima Reimpresión (2001)

Expósito, J. \& Manzano, B. Escuela TIC 2.0: aprendizaje del alumnado de primaria en contextos educativos y socio familiares. EDUTEC, Revista Electrónica de Tecnología Educativa, 45. Recuperado el 13//01/2016 de http://edutec.rediris.es/Revelec2/Revelec45/escuela_TIC_aprendizaje_contexto_educativo_sociofamiliar. html. (2013)

Ford, A. Modelling the environment. An Introduction to system dynamics. Models of environmental systems. Island Press. Washington DC (1999)

Forrester J.; Road Maps: A guide to learning system dynamics. Boston: System Dynamic Group, Sloan School of Management, MIT, the United States of America (1997) 
Gobernación de La Guajira. Boletín de prensa No. 34. Marzo 16 de 2015. http://www.laguajira.gov.co. (2015)

González Rodríguez, O. Uso y tratamiento del Wayunaiki en dos instituciones etnoeducativa wayuu (Doctoral dissertation) (2015)

Gómez, U E; Andrade, H y Vásquez, C A. Lineamientos Metodológicos para construir Ambientes de Aprendizaje en Sistemas Productivos Agropecuarios soportados en Dinámica de Sistemas. Inf. tecnol. [Online]., vol.26, n.4 [citado 2016-01-22], pp. 125-136 http://dx.doi.org/10.4067/S071807642015000400016. Disponible en: <http://www.scielo.cl/scielo.php?script=sci_arttext\&pid=S071807642015000400016\&lng=es\&nrm=iso >. ISSN 0718-0764.(2015)

Guibourg R, Ghigliani M., Guarinoni A y Ricardo V. Introducción al Conocimiento Científico Editorial, 60-75. Universitaria de Buenos Aires, (1985)

IDEAM. Instituto de Hidrología, Meteorología y Estudios ambientales de Colombia. Vulnerabilidad del Departamento de La Guajira por el fenómeno del niño. Informe en la Página http://goo.gl/PCyrt4 (2014)

Jiménez R., M. Aproximación teórica de la exclusión social: complejidad e imprecisión del término. Consecuencias para el ámbito educativo Estudios Pedagógicos XXXIV, № 1: 173-186, (2008)

Jiménez, E., Reclade, L., y Silva, M., Visión comparativa entre redes de Petri continuas y diagramas de Forrester. XXIII jornadas de automática. (2002)

Mangin, L, JP, Mallou V. J. Modelización con estructura de covarianza en Ciencias sociales. 145-175 Gesbiblo S.L. España, (2006)

Tamayo, M. El proceso de la investigación científica.4ta, Edición. México Limusa.pp 13-44 (2004)

Martínez C., P.C. El método de estudio de caso Estrategia metodológica de la investigación científica Pensamiento y gestión, № 20 ISSN 1657-6276 (2006)

Morín, E, Sánchez A. La ecología de la civilización técnica. De la noción de medio técnico a ecosistema social. Universitat de Valencia. (1981)

López N, Sandoval I. Métodos y Técnicas de investigación cualitativa y cuantitativa. Documento de Trabajo. Sistema de Universidad Virtual, 3-23. Universidad de Guadalajara. (2006)

Ricoy, M. C., Feliz, T. y Sevillano, Mạ . L., Competencias para la utilización de las herramientas digitales en la Sociedad de la Información. Educación XX1, 13 (1); 199- 219 (2010) 